\title{
Flexural and impact properties of biopolymer derived from sugar palm tree
}

\begin{abstract}
The effect of glycerol concentration $(15 \mathrm{w} / \mathrm{w} \%, 20 \mathrm{w} / \mathrm{w} \%, 30 \mathrm{w} / \mathrm{w} \%$ and $40 \mathrm{w} / \mathrm{w} \%)$ to the flexural and impact properties of plasticized sugar palm starch (SPS) was investigated in this present paper. Prior to the testing, the sugar palm starch extracted from the interior part of sugar palm stem was mixed with common glycerol (was used as a plasticizer) to form a novel biopolymer. The flexural and impact test were carried out according to ASTM D790 and ASTM 256 respectively. From this investigation, it is found that the $30 \%$ glycerol concentrated SPS biopolymer showed the highest flexural strength and flexural modulus with the value of 0.13 MPa and 87.54 MPa respectively. For the impact analysis, it is also found that same biopolymer showed the highest impact strength which is $6.13 \mathrm{~kJ} / \mathrm{m} 2$.
\end{abstract}

Keyword: Biopolymer; Sugar palm starch; Glycerol; Flexural; Impact 